\title{
MODELO DE QUÍNTUPLE HÉLICE EN LA GENERACIÓN DE EJES ESTRATÉGICOS DURANTE Y POSTPANDEMIA 2020
}

\section{FIVE-HELIX MODEL IN THE GENERATION OF STRATEGIC AXES DURING AN AFTER 2020}

Mawency Vergel Ortega ${ }^{1}$

Olga Marina Vega ${ }^{2}$

Victor Jhoel Bustos Burbano ${ }^{3}$

\section{RESUMEN}

La investigación tiene como propósito validar el modelo de quíntuple hélice identificando ejes estratégicos como factores que influyen la formación de los estudiantes de la universidad Francisco de Paula Santander-CúcutaColombia, de acuerdo a su perfil, percepciones y necesidades; para este estudio se realizó una encuesta en la población total de estudiantes matriculados en I-2020, así como egresados, empresarios externos. La metodología estudio

1 Departamento de Matemáticas y Estadística, Facultad de Ciencias Bàsicas, Universidad Francisco de Paula Santander, Cúcuta, Colombia. mawencyvergel@ ufps.edu.co https://orcid.org/0000-0001-8285-2968

$2 \quad$ Programa de Enfermeria., Facultad de Ciencias de la salud, Universidad Francisco de Paula Santander, Cúcuta, Colombia. olgavega@ufps.edu.co https://orcid. org/0000-0002-5525-0088

$3 \quad$ Facultad de Ingeniería, Universidad Francisco de Paula Santander, Cúcuta, Colombia. jhoelbustos@ufps. edu.co, https://orcid.org/0000-0001-6954-2260 descriptivo, de análisis de contenido. Resultado: En el marco de la emergencia sanitaria generada por el Covid surgen tres categorías emergentes considerados como ejes estratégicos: generación de cadena de valor, saber y generación de conocimiento; Identidad y Universidad circular para la comunidad, moderna e inteligente; con subejes Universidad con gestión de calidad en la formación, universidad con gestiòn investigadora, productiva e innovadora, universidad sustentable, resiliente con equidad y hábitat de calidad, universidad multicultural con gestiòn para la convivencia, protección del ser humano, la paz, el deporte y bienestar; con gestiòn en redes, disruptiva, competitiva e internacional; categorías fenomenológicas presentes son equidad, sostenibilidad, global, integrada e inclusiva. 
PALABRAS CLAVE: Educación superior, programas, sesiones remotas, perfiles.

\section{ABSTRACT}

The purpose of the research is to validate the five-fold helix model by identifying strategic axes as factors that influence the training of students at the Francisco de Paula SantanderCúcuta-Colombia University, according to their profile, perceptions and needs. For this study, a survey was conducted on the total population of students enrolled in I-2020, as well as graduates and external entrepreneurs. The methodology is based on a mixed, descriptive and content analysis approach. Result: Emerging categories considered as major strategic axes generation of value chain with knowledge and knowledge generation, Identity and, circular university, for the community, modern and intelligent, with sub axes University with quality management in the formation towards the excellence, university with research, productive and innovative management, sustainable university, resilient with equity and quality habitat, multicultural university with management for the coexistence, the protection of the human being, the peace, the sport and the well-being; university with management in networks, disruptive, competitive and international, phenomenological categories present are equity, sustainability, global, integrated and inclusive.

KEYWORDS: higher education, undergraduate, academics, virtuality.

\section{INTRODUCCIÓN}

Al imponerse la innovación como factor clave para el desarrollo, la sostenibilidad y los escenarios de cooperación (Vergel, 2018), se implementan en el mundo modelos como triple hélice (González, 2019, Acuña, 2014, Arboledas, 2010). La Universidad Francisco de Paula Santander, apuesta por un modelo de quíntuple hélice como motor y generador de energía para el cumplimiento de su misión y visión en el periódo de transición y alternancia para ser una Universidad, adaptada y localizada en la gestión, articulada a Objetivos de Desarrollo Sostenible (Gallardo, Vergel, 2019), a políticas, planes internacionales, nacionales, gubernamentales, del Ministerio de Educación Nacional, del Ministerio de ciencia tecnología e innovación, del Ministerio TIC, de la Misión de sabios y normativas departamentales que potencia el desarrollo educativo regional y visionan la universidad al futuro.-En la Universidad Francisco de Paula Santander se armonizan los procesos académicos (Vergel, 2015); de investigación, innovación, inclusión, extensión y proyección social (ASCUN, 2010), desde ambientes presenciales, articulados y apoyados en tecnologías de la comunicación hacia lo híbrido con sustento en la tecnología; así como los procesos de apoyo administrativo, clima y bienestar acorde a la realidad pasada, presente y futura; realidad que impulsa a la generación de conocimiento orientado en la innovación y la calidad de procesos de enseñanza/aprendizaje inmersos en su tendencia curricular digital, dentro del enfoque dialógico crítico, donde prima la investigación, el diálogo, la transferencia de conocimiento, el emprendimiento y el fomento de la cultura, con una clara vocación de intercambio y cooperación; un futuro comprometido con la acreditación y responsabilidad con su comunidad, el desarrollo sostenible y sustentable. La información fue complementada con investigaciones similares, con validez y fiabilidad estadística. En el marco de escenarios donde priman los escenarios de asistencia remota, en la Universidad se busca mantener la calidad de sus programas y gestiòn, sus índices positivos, lo cual hace necesaria una ajuste a políticas institucionales, para dar respuesta a políticas nacionales e internacionales, necesidades de formación de los jóvenes, competencias, resultados de aprendizaje, acorde al perfil del futuro profesional. El escenario conlleva a priorizar 
la visión de futuro asociada a una universidad "innovadora, tolerante, creativa, tecnológica y con talento" en la línea de los objetivos de desarrollo 2030 (ODS, 2019).

\section{METODOLOGÍA}

Se realizó un estudio exploratorio-descriptivo con la población total activa, estudiantes de pregrado y muestra no probabilística de profesores, directivos e integrantes del sector productivo. Se diseña modelo de desarrollo de cinco hélices (Figura 2) basado en el modelo de desarrollo virtuoso modelo que tiene como primer hélice la universidad constituida por toda su comunidad académica $(U)$, como segunda el gobierno y organizaciones del ámbito mundial, nacional y regional $(G)$, una tercera hélice la constituyen las empresa e industrias (E), una cuarta hélice la sociedad (S) y una quinta hélice las redes académicas $(R), y$, de investigación de apoyo e instituciones que generan sinergias y trabajo colaborativo en pro del crecimiento sostenible, incluyente basado en el conocimiento para el desarrollo de la sociedad. La primera fase se ha centrado en la revisión de bases estratégicas, desde metodologías de marco lógico, seis sigma, y su transposición a la matriz Came (Huelva, 2019). La segunda fase, ha consistido en la elaboración de propuestas estratégicas en el marco de estrategias ofensivas considerando las fortalezas y emplearlas con las oportunidades del contexto y situacionales de emergencia sanitaria, siendo consideradas como estrategias ofensivas, de supervivencia implican combinar las debilidades internas con las amenazas del entorno para definir las estrategias de supervivencia que afronta para superarlas; estrategias defensivas, combinan las fortalezas junto con las amenazas del entorno que deberán ser mantenidas a lo largo del tiempo. Se trata, de mantener las fortalezas frente a amenazas externas, estrategias de reorientación, basadas en las debilidades, junto con la aparición de nuevas oportunidades del entorno, para reorientar de forma positiva a la universidad, ajustar/actualizar/convertir. Tomando como base lo establecido en la Fase II, se continùa con el desarrollo de los niveles: Ciclo de construcción que relaciona estrategias de reorientación, supervivencia, ofensivas, defensivas junto a propuesta temporal de propósito, identificación de valores, selección de ejes, planes y políticas nacionales e internacionales, la generación de modelo de cinco hélices para identificar $y$ definir la Estrategia transversal, identificación de ejes estratégicos, los cuales representan la categoría máxima dentro del Plan institucional y detallan grandes áreas de actuación de la universidad durante su vigencia; creaciòn de objetivos estratégicos: describen, para cada eje, las intenciones específicas de la universidad, incluyendo aquellos aspectos que la universidad necesita lograr para llevar a cabo el plan estratégico. Se definen las líneas estratégicas referidas al conjunto de actuaciones que definen el actuar para alcanzar los objetivos estratégicos durante la vigencia del plan, planes de actuación concretas a llevar a cabo para desarrollarlo. La tercera fase, la participación pública. Se diseña la inserción de estructuras participativas a través de anàlisis de contenido, encuesta de opinión, satisfacción y análisis de acciones implementadas en el marco del I-2020 en contextos propiciados por la pandemia, de manera que se permita una planificación estratégica en la cual participe toda la comunidad académica, que conlleve a continuar liderazgo de la rectoría con visión estratégica y capacidad de gestión (Vergel, 2015), a fin de propiciar la toma de decisiones y alcance de objetivos, construcción propiciadora de la autogestión (Bernal, 2019) de emociones de la comunidad.

\section{RESULTADOS}

Desde la misión de sabios (Ministerio de Ciencia, 2020), se proponen como retos una Colombia productiva sostenible, una Colombia Biodiversa y una Colombia equitativa, se retoman sus 
ejes y propósitos en Biotecnologia- ciencias básicas-industrias creativas y culturalesdesarrollo con equidad- oceanos y recursos hidrobiologicos-tecnologias convergentespensamientos laterales, diversidad e intración de conocimientos y participación del sector productivo como apuesta a convertir en bienes $y$ serios parte del conocimiento para mejorar el PIB de $0.61 \%$ desde la horizontalidad construyendo desde las regiones y sus particularidades, en una ruta territorial de eje fundamental para la democratización del conocimiento con acciones locales para construir apuestas colectivas desde la universidad. Eliminar barreras, deconstruyendo imaginarios, tanto culturales como para construir una academia fuerte para generar valor agregado donde el conocimiento básico se convierta en elemento que proporciones soluciones y vida. El Plan de Desarollo Departamental presenta como programas La educaciòn con calidad para transformar vidas, educación con calidad para reducir brechas y dentro de sus ejes el Bienestar, Habitat, convivencia, Gobernanza, infraestructura y productividad.

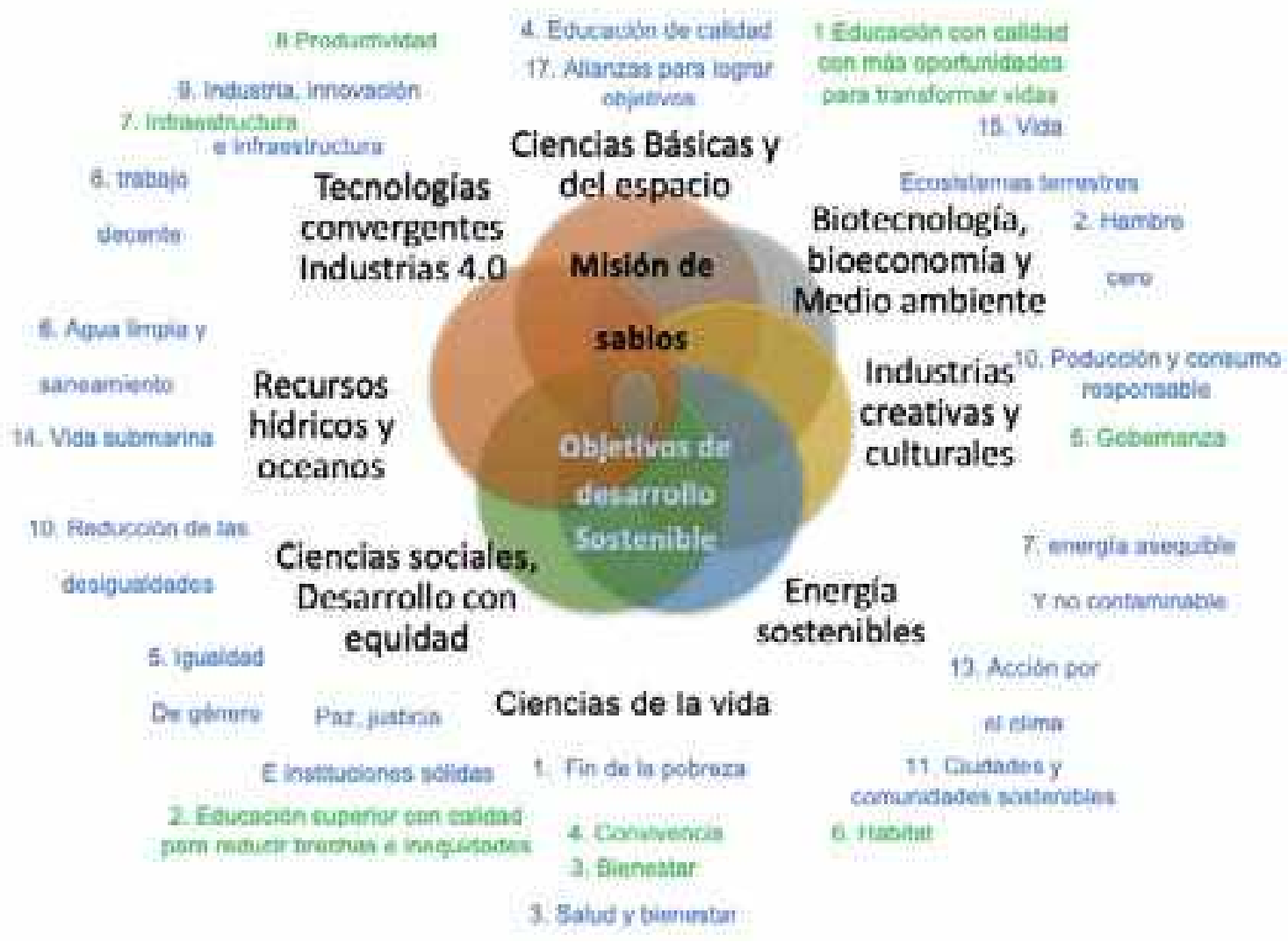

Figura 1. Ejes, lìneas y programas a vincular. Fuente: Adaptado ODS(2019), Ministerio de Ciencia, Tecnologìa e Innovaciòn(2019). Gobernaciòn de Norte de Santander (2020). 


\section{RESULTADO PLANIFICACIÒN Y JERARQUIZACIÒN NIVEL 1}

Objetivos de diseño se orientan a una gestión eficiente y transparente de su talento humano y de sus recursos. Una universidad con talento humano con capacidades, competencias y habilidades para la formación de los futuros profesionales de la región en escenarios presenciales, semipresenciales 0 totalmente mediados por las tecnologías de la información y la comunicación, para lo cual establece planes de formación y capacitación del talento humano, así como la cultura de la capacitación en sus estudiantes. Para la comunidad académica estudiantes y profesores representan el corazòn y motor de la Misión Institucional. Los propósitos en escenarios de transición se centran entonces en la diferenciación de la Universidad, con enfoque hacia la calidad, investigación e innovación en problemáticas de la región, nación y aquellas que permitan soluciones de diferentes gobiernos; haciendo hincapié en ejes estratégicos y estructuras de su modelo de estrategia; una universidad generadora y potenciadora de la transferencia de conocimiento.

La oferta con calidad académica, impulsada por la igualdad, busca la adaptación de la comunidad académica a escenarios donde la era digital permiten la transición desde el apoyo mediado por la tecnologías de la comunicación y la semipresencialidad; la concientización de la protección de la salud y la cultura del autocuidado y la cultura de cuidado del medio ambiente. Esta adaptación a la tecnología, medios y herramientas busca la generación de gestión de contenidos, desde la transversalidad del currículo acorde a perfiles de formación en cada programa académico en pregrado y postgrado que oferta la universidad. Objetivos orientados a definir zonas de uso en la infraestructura del campus de la universidad, que otorguen seguridad a la comunidad, conllevan a establecer un plan de ubicación de recursos, señalización de espacios y zonas de tránsito, aulas, laboratorios, parqueaderos, con el objeto de establecer distancias adecuadas entre la comunidad; así como a la planeación y programación adecuada de horarios en cada programa académico y la generación de protocolos y plan de comunicación correspondiente. El Liderazgo, interviniente y modelo organizativo de gobernanza requiere incorporar la perspectiva de los diferentes organizaciones, ministerios, sectores, redes; vicerectorìas, jefaturas y unidades, abarcando los niveles administrativos del Estado, comunidades y entidades locales como gestoras últimas de muchas de las actividades que inciden directamente en la economía circular, así como los agentes sociales, la comunidad académica, los ciudadanos, cuyas decisiones, así como su compromiso con la calidad educativa son fundamentales. - La Cadena de valor UFPS son las Políticas institucionales. Madurez: Modelo de madurez de Gartner- Universidad hacia madurez. Resultado Mejora Índice de programas sube de 67 a 78.4, lo cual significa en un avance en un nivel medio a alto.

\section{Caracterización general comunidad} población académica corresponde a 16700 estudiantes adulto-joven con alto sentido de pertinencia institucional, seleccionan la ufps por su posicionamiento en la regiòn y liderazgo de egresados, son usuarios con alta satisfacciòn de plataforma PLAD; dependiente de padres $(80 \%)$, acuden a entidades para crédito bancario $(54.1 \%)$, apoyo a través de programas como jóvenes en acción (78\%), estímulos académicos (35\%), comunidades o apoyo a víctimas (31\%); de otra parte, manifiestan necesidades que giran en torno ejes innovación en calidad educativa, emprendimiento, tic. Actualmente la Universidad, cuenta con 838 profesores; 79 con formaciòn doctorado, 5 postdoctorado, 420 magister; 344 especialistas; 539 menores de 50 años. El análisis de clasificación permite 
observar alta asociación entre variables con variable predictiva Ingreso a plataforma de apoyo académico Plad y variables de incidencia departamento acadèmico y programa, con tendencia a valoración positiva del profesor y del aprendizaje, tenencia alta de herramientas de los profesores, media de estudiantes, y alto uso de herramientas didàcticas. Resultados de las pruebas Saber Pro (Bedoya, 2011), en 2019 muestra resultados dentro del promedio nacional.

Resultados Entrevista profesores y egresados señalan como Fortalezas: 1. Le identifican como una institución prestigiosa referente y reconocida regional, nacional con algunos avances en reconocimiento internacional. Capacitada para contribuir en una sociedad diversa, dispone de una oferta de programas de calidad (pregrado, maestrìa y un Doctorado). Competitiva, orientada a la formación integral y permanente (apoyada en el conocimiento abierto incorporando dos plataformas on-line), que facilita ingreso, continuidad, pràcticas pedagògicas, apoyo a inserción laboral, la flexibilidad y la movilidad, permite una gobernanza eficiente en el cumplimiento de su misión, basada en los objetivos estratégicos establecidos por la comunidad universitaria, la utilización efectiva de los recursos y la adecuada gestión de calidad de los servicios. Las dinámicas de la institución proporcionan un sistema motivador de desarrollo humano y se dispone de personal comprometido, competente y eficaz, organizada y reconocida. Estudiantes manifiestan una actitud participativa, motivados por el auto-aprendizaje y la superación. Egresados tienen una formación integra, ocupan cargos adecuados a sus competencias y capacitados para afrontar las necesidades del desarrollo, crecimiento económico, investigativo y cultural de la sociedad 3. La investigación está alineada con la política de investigación, objetivos de desarrollo sostenible, metas del Ministerio de Ciencia, tecnología e innovación, es reconocida a nivel nacional, transfiere el conocimiento y además es un motor del desarrollo sostenible de su entorno económico y social. La relación universidad, sociedad y empresa es fluida integrada a través de SIES +, y se proyecta proactivamente sobre su entorno social. 4. Dispone de espacios, infraestructura y servicios adecuados, sostenibles y gestionados eficientemente para toda la comunidad universitaria, especialmente de aquellos que son necesarios para la docencia, investigación, innovación y transferencia. 5. Se producen algunos intercambios entre estudiantes, con universidades y centros de investigación de prestigio a nivel global. Se han establecido y consolidado redes de colaboración nacional e internacional en docencia, investigación y cooperación, que permiten la creación y promoción de proyectos compartidos en los distintos campos. 6. Es una institución socialmente responsable que respeta los principios éticos y está comprometida con el desarrollo sostenible y la promoción de los valores ciudadanos. 7. Dispone de la financiación suficiente para realizar sus funciones, por la captación de recursos del gobierno nacional, gestionados de forma eficiente, equitativa y transparente. 8. servicios digitales le ha permitido racionalizar procedimientos y optimizar recursos. La comunicación interna se apoya en las tecnologías y es instrumento de la comunidad universitaria. 9. Es una universidad con bienestar, saludable, dispone de un entorno de trabajo inclusivo, equitativo. La igualdad de oportunidades entre mujeres y hombres es una realidad. 10. La comunidad académica està consolidada. Profesores, estudiantes, padres, egresados mantienen sus lazos con la institución, proyectan su imagen, y son sus mejores representantes. Se apoya en un observatorio de empleabilidad con el objetivo de establecer nexos continuos con egresados, potenciar su proyecciòn laboral, proyectar demandas, hacia donde se encamina el mercado laboral y cuáles 
van a ser los retos del futuro, para encaminar la oferta, docencia y la investigación.

\section{Debilidades a transformar a corto plazo:} necesidad en equipamiento, datos, internet estudiantes, Formación, capacitación, problemas con la calidad pedagógica y metodológica (15 profesores), ausencia herramientas en todas las asignaturas, Deficientes habilidades en TIC 10 profesores, debilidad en gestión de contenido, gestión de comunicación, horario docente; control medidas de mitigación prevención de contagio. Oportunidades a explotar: políticas, acompañamiento plan padrino, plataformas académicas gratuitas, competencia, motivación, tendencias favorables, capacitación, recurso humano, trabajo colaborativo, avance en gestión de contenido de algunos docentes; internacionalizaciòn, movilidad a travès de virtualidad, redes. Fortalezas vs oportunidades a mantener: gestión alta dirección, políticas institucionales, organización, equipo talento humano, buen ambiente laboral, calidad de producto final, posibilidades gestión donaciones o aportes gobierno-Departamento-Municipio, posibilidades de financiación de proyectos, experiencia de recursos humanos, estímulo, creatividad talento humano. Oportunidaddebilidad: plan padrino-plataforma gratuita para capacitar. Amenazas a afrontar: Cambios en la legislación, deserción, Competencia agresiva, tiempo en desarrollo tecnológico y científico, gestiòn recurso financiero. Propòsito: Posicionarse nacional e internacionalmente como una institución pública de educación superior con programas académicos de alta calidad transpormadores de la sociedad $y$ petenciadores de la innovaciòn, constituyendose en una universidad incluyente y socialmente responsable. Planificaciòn temporal. En el marco de la emergencia santaria generada por el Covid surgen tres grandes Familias de categorias emergentes considerados como grandes ejes estratègicos

\section{GRANDES EJES ESTRATÉGICOS QUE SURGEN DE SUS FORTALEZAS + OPORTUNIDADES}

Gran eje 1 generación de cadena de valor. El saber, la generación de conocimiento constituyen su esencia y elemento que gira en torno a su comunidad académica y que articula todas las acciones. Su fin y visión proyectan el generar, transformar, transferir conocimiento en todas las ciencias en continua mejora y conexión con la comunidad y contexto.

Gran eje 2 identidades. 58 años de la UFPS dan identidad forjada por la experiencia que da soporte para su desarrollo, sostenibilidad y sustentabilidad futura reafirmando su compromiso de transformar vidas en su entorno, contextos proyectándose a la sociedad.

Gran eje 3. Universidad circular, para la comunidad, Moderna e inteligente. Enfrentar nuevos retos que le configuren como universidad creadora y transformadora de conocimiento con el apoyo de cada integrante acorde a una estructura organizacional dinámica y eficiente, que permite el logro y alcance de sus objetivos, retos $y$ fines.

Categorìas dentro de estos ejes dan lugar a ocho Líneas Principales de Actuación, Cinco de ellas relacionadas a una universidad círcular: Formaciòn, Oferta, efectividad en la Gestión, programas, y, recursos y sustentabilidad financiera, y las tres restantes, con carácter transversal: Sensibilización y Participación, Investigación, Innovación y Competitividad, y, empleo y perfil de Formación del egresado.

En el marco de entrevista con items asociados a solución de problemas del ambito social emergen categorìs agrupadas como Políticas Clave de: Calidad, Tecnologias de la informaciòn y comunicación, Propiedad intelectual, Investigación e innovación $(I+D+i)$, responsabilidad social, de igualdad, seguridad 
de la información, Política financiera-económica, Economìa circular, Politica ambiental y de sostenibilidad, Empleo seguimiento y perfil de formación egresados, Oferta, Política RetenciònDeserciòn, Relaciones y Sinergias Institucionale, Internacionalizaciòn, Riesgos, Formación en lenguas, Bienestar, cultura y deporte, proyecciòn social, proyectos interinstitucionales.

subEjes transversales estratégicos que surgen de Debilidades + Oportunidades + Amenaza + fortalezas logradas y a fortalecer de plan de desarrollo 2011-2019

Implementado el modelo de quíntuple hèlice, resultado de triangulación de métodos, análisis de contenido, se consideran dentro de la investigación, subejes centrales para el plan de la universidad Francisco de Paula Santander, 1. la universidad con gestión de calidad en la formación hacia la formación de excelencia, 2.la universidad congestión en redes disruptiva, competitiva e internacional; 3 . la universidad multicultural con gestión para la convivencia la protección del ser humano, la paz, e deporte y el bienestar; 4.la universidad sustentable, resiliente, con equidad y habitat de calidad y con eje transversal 5 . la universidad con gestion investigadora, productiva e innovación disruptiva (Figura 1). Sus valores estratégicos centrados en equidad, calidad, sustentabilidad, integración e inclusión.

El ámbito del eje 1. proporcionar una oferta formativa de calidad, amplia y que potencie la empleabilidad de egresados e inserción laboral de estudiantes, una institución comprometida con la creación, transmisión y transferencia de conocimiento, foco de atracción para el personal docente-investigador y para personal administrativo, en busca de oferta de doble titulaciòn institucionales que permitan establecer sinergias, incrementar además la movilidad de estudiantes, profesores y promover redes docentes y de investigación entre las universidades.

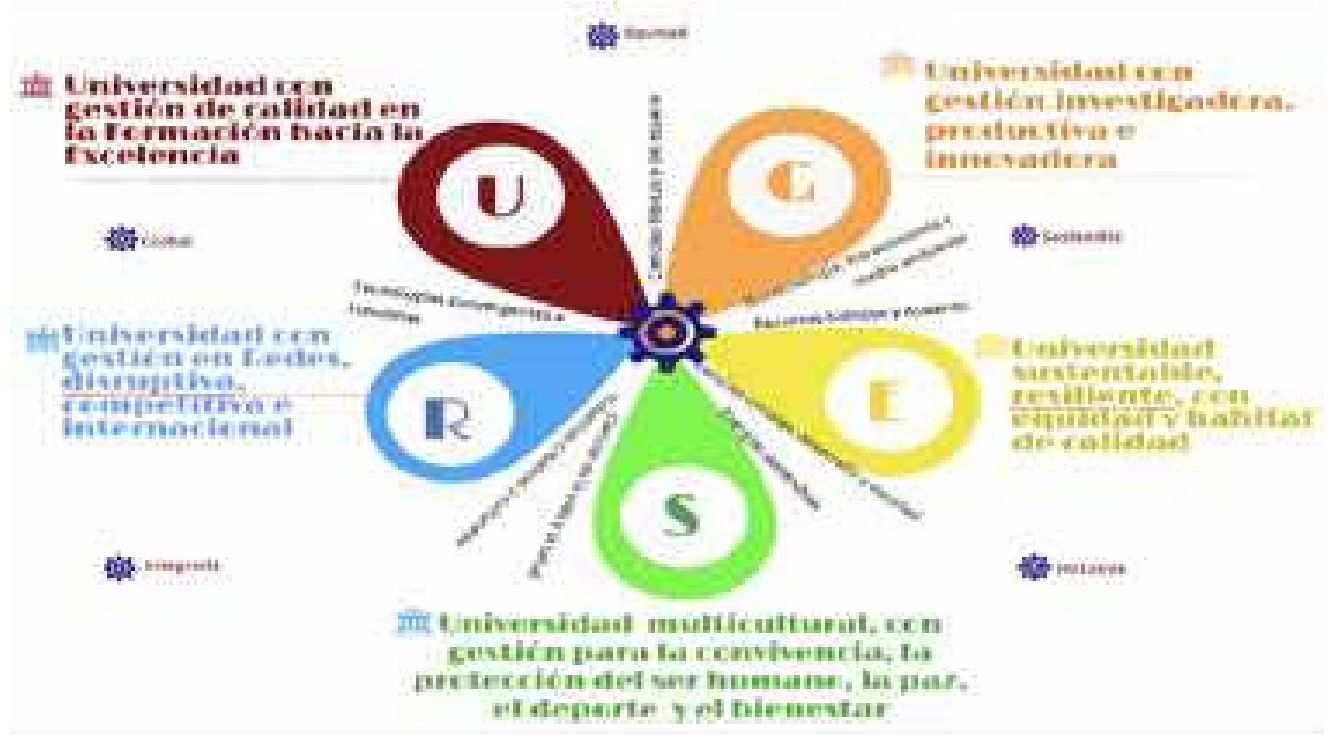

Figura 1 Propuesta Ejes estratégicos modelo de 5 hélices. Fuente: Vergel (2020) 
Propiciar innovación y emprendimiento en la comunidad universitaria a través de los elementos que definen la cultura organizativa, para ello, identificar y favorecer pràcticas, comportamiento innovador emprendedor entre investigadores, estudiantes y egresados que pueda derivar en creación de empresas, mejorar el perfil competencial de estudiantes y egresados y realizar seguimiento del nivel de empleabilidad para incorporarse a organizaciones basadas en la innovación a traves de programas como emprendeTic. La evolución de la universidad hacia la innovación y el emprendimiento suma el identificar la función de la creación de empresas por parte de estudiantes y egresados de la universidad a partir de Conocer el potencial de mercado que tienen los resultados de la investigación producida por los grupos de investigación de la Universidad

Son objetivos- Fortalecer y construir una oferta formativa en educación superior y cultural que responda a la demanda de los retos sociales que fomente la transversalidad de programas académicos ofertados basados en la complementariedad e incremente la relación oferta realidad social, potencie el enfoque aprendizaje-transformación- proactividadservicio; la competencia digital; potencie la oferta formativa complementaria a partir de resultados de aprendizaje; promueva la generación y transmisión cultural y la actividad deportiva, el autocuidado incorporando el conocimiento generado de investigaciones y propiedad intelectual de la comundad acadèmica. -Impulsar la obtención de acreditación de Calidad, registros y sellos de calidad (internacionales) de reconocido prestigio nacional e internacional. -impulsar a través de reingeniería programas de maestría y doctorado de profundización e investigación para alcanzar la excelencia. -Fortalecer el uso de las TIC en el Sistema de Gestión Académica, administrativa, investigativa, de innovaciòn y de Prevención de Riesgos. O subeje : Desarrollar una gestión ágil, sostenible, eficaz y eficiente de los recursos a través de aplicación de las tecnologias de la innovaciòn. O subjeje: Crear y desarrollar un plan de gestión y mantenimiento de las infraestructuras tecnològicas y de comunicaciòn

En eje 2 subeje Universidad sustentable: -Desarrollo normativo e-administración, Fomentar captación de fondos, -Promover gestión eficiente económico-financiera, Gestionar el entorno natural -Mantener coeficientes de edificabilidad del campus, -Gestionar la accesibilidad y movilidad sostenible, Gestionar la contaminación atmosférica. -Gestionar un modelo de espacio de densidad y distribuciòn de personas - Incrementar y mejorar la comunicación institucional interna y externaFomentar la colaboración con asociaciones empresariales. El eje 3 con el subeje Universidad con gestiòn de redes, disruptiva, competitiva e internacional, desarrollo, actualización e implantación de estrategias institucionales de internacionalización. La internacionalización considerada como una dimensión horizontal que afecta a toda la estructura y procesos de la universidad.

El plan contempla como objetivos el Consolidar una universidad fuerte $e$ internacionalmente atractiva que promueva la movilidad de entrada y salida de los mejores estudiantes, profesores, investigadores y personal de administración y servicios, la calidad educativa, el potencial del segundo idioma para la educación superior, la internacionalización de los programas formativos y las actividades de I+D+i, contribuyendo a la mejora y competitividad nacional e inmersiòn en el ambito internacional, así como al desarrollo socioeconómico de su entorno próximo, basado en el conocimiento. Objetivos Subeje 2: Potenciar y establecer acuerdos e intercambio con universidades, redes y organizaciones; establecer convenios de movilidad latinoamericana y europea con países que han 
incorporado egresados, de fuerte demanda (Brasil, Mèxico, Panamà, Australia, Alemania, otros); Fomentar la internacionalización de programas de pregrado, maestrìa y doctorado; Incrementar las acciones de promoción, y diseñar maestrias con participación de empresas e instituciones externas, doctorados y másteres interuniversitarios; Promover la innovación, cultura emprendedora y creativa.

Eje 2 subeje 1 y 3 Universidad multicultural con gestiòn para la convivencia, la paz, la protecciòn del ser humano, la paz, el deporte y bienestar. Objetivos: Maximizar la eficacia y la eficiencia en el uso de las instalaciones deportivas para toda la comunidad universitaria; desarrollar el Plan Integral de Deporte; Implementar la perspectiva de inclusiòn, y género en la estructura de programas y proyectos en el ámbito de la cultura, la actividad física y del deporte; Fomentar la extensión universitaria en municipios a partir de la consolidaciòn de redes universitarias, investigaciòn y entre organizaciones.; Evaluar y estimular, a través de la cultura, la convivencia y el deporte, valores de pluralismo, igualdad, integración social, tolerancia y espíritu crítico para toda la comunidad universitaria y su proyección a la sociedad; Dinamizar cultura de la paz y en Subeje 5 . En investigaciòn Diseñar e implantar un Plan de marketing para prestación de servicios a externos

Retos a corto y mediano plazo. A fin de dar respuesta a situaciones que enfrenta la humanidad y la universidad en el ser sostenible, integrada, global, que privilegia la equidad e igualdad:-Garantizar que la evolución de la matrícula no afecte la gobernabilidad institucional así como mantenerla matricula asegurando altos estándares de calidad; Actualizar modelo educativo a fin de elevar los niveles de eficiencia terminal por cohorte generacional en la oferta; Establecer modelo de Formación, capacitaciòn e instrucción del talento humano en diseño, creación y gestión de contenido, evaluación y procesos de enseñanza aprendizaje para apoyar procesos de aprendizaje a través de TIC; Establecer un modelo de evaluación de profesores integral, sostenible, que potencie la mejora de la calidad docente; Establecer un modelo de gobernanza, gestiòn de tecnologìas $y$ de seguridad de la informaciòn a travès de un sistema de seguridad de la informaciòn; Analizar continuamente resultados de la evaluación externa para considerarlos en los procesos de reflexión y toma de decisiones para la mejora continua y el aseguramiento de la calidad; Contribuir de manera oportuna a elevar los niveles de bienestar de la sociedad, el crecimiento económico, la productividad y capacidades de innovación, a través de aportaciones relevantes, pertinentes, y con altos estándares de calidad; Dinamizar programas y planes en cultura, los valores y la participación ciudadana mediante programas culturales y de salud que generen cohesión social, respeto y equidad; Gestionar y optimizar plataforma de gestión de la información; Gestionar procesos de admisión de estudiantes II-2020; Gestionar procesos de Permanencia y graduación; Gestionar procedimientos de archivo en la nube; Alcanzar altos nivel de logro de estudiantes; Consolidar la investigación, desarrollo tecnológico, innovación y cultura en los programas acadèmicos; Asegurar equilibrio en dedicación profesoresfunción, reconocimiento-perfil, incremento en doctorados y posicionamiento de Investigadores en Colciencias.

Acciones a corto plazo A1: Diseñar e Implementar Plan de formación, capacitación de talento humano en competencias digitales transversales, pràcticas, evaluación. A2: Diseñar cursos de interés estratégico con metodología MOOC, blended, aplicativos, software/ herramientas didàcticas A3: implementar Plan de Admisión Permanencia Graduación. A4: Desarrollar el empleo de técnicas analíticas de macrodatos. A5: Optimizar Infraestructura 
tecnológica con filosofía de diseño centrada en usuario. Automatizar procesos en formación,investigación, transferencia y gestión. A6: Actualizar plataforma PLAD para docencia en línea A7: Crear y desarrollar plan de apoyo para la producción de contenidos digitales. A8: Ajustar plan de inversiones (Gestión gobierno uso de recursos). A9: Diseñar e implementar programas para apoyo estudiantes con necesidades manifiestas A10: Diseñar y crear plan de Infraestructura y adecuación de aulas. A11. integrar tecnologías en la mejora de la movilidad, eficiencia energética, explotación eficiente y colectiva de las infraestructura. A12: Diseñar e implementar plan de Seguridad, gestión de riesgo, Laboratorios regreso Aulas y campus 1-2021 protocolos bioseguridad UFPS para la alternancia. A14: Diseñar plan de salud, carga de trabajo y evaluación emocional del talento humano. A15: Diseño de politicas y Acuerdos fomento permanencia y admisiòn en epoca de emergencia sanitaria y formaciòn a travès de actividad sincrònica o hìbrida. A16: reingenierìa a procesos de documentación de servicios TI (capacidad, disponibilidad, continuidad, seguridad de la informaciòn y proveedores) (Figura 3) A17. Crear web "Futuro Estudiante" que, proporcione información clara y atrayente a futuros estudiantes sobre procedimientos y tràmites necesarias para el acceso a la universidad, además de valuar $y$ ofrecer orientación hacia programas que se ajusten a perfil y capacidades. A18. Mejorar posibilidades de acceso a la información y a los servicios por parte de la comunidad universitaria mediante el desarrollo de aplicaciones móviles A19. mejorar la experiencia de los usuarios a través de interfaces más centradas en ellos. A20. Planificar la utilización de espacios e infraestructuras de tal manera que sea posible un uso más eficaz, eficiente y transversal, que los ponga al servicio de las necesidades de la comunidad universitaria y de la sociedad. Desarrollar el proyecto rutas saludables, Espacios protegidos y movilidad sostenible. Adecuar los espacios docentes y escenarios de pràctica a las necesidades actuales. A21. Mejorar la divulgación de los resultados de investigación e innovaciòn de la Universidad. A22. transformación digital del trabajo (digitalizar procesos involucrados en formación, investigación y transformación del conocimiento) y el empleo de técnicas de análisis de macrodatos (big data) como herramienta de ayuda a la toma de decisiones.

A mediano Plazo A22: Actualizar Proyecto educativo institucional acorde a politicas nacionales, tendencia curricular y realizar ajustes curriculares en torno a resultados de aprendizaje. A23: Aumentar prácticas internacionales A24. Crear y desarrollar el plan de implantación de la e-Administración A25. Elaborar y desarrollar un proyecto de reutilización de información A26. Iniciar creaciòn de normativa para la implantación de dobles titulaciones A27. Incrementar el número de doctorado y maestrìas propias e interuniversitarios A28. Fomento líneas doctorado interdisciplinares A29. Redefinir y optimizar estructura organizacional A30. Incrementar captación de fondos del sector privado para la investigación 


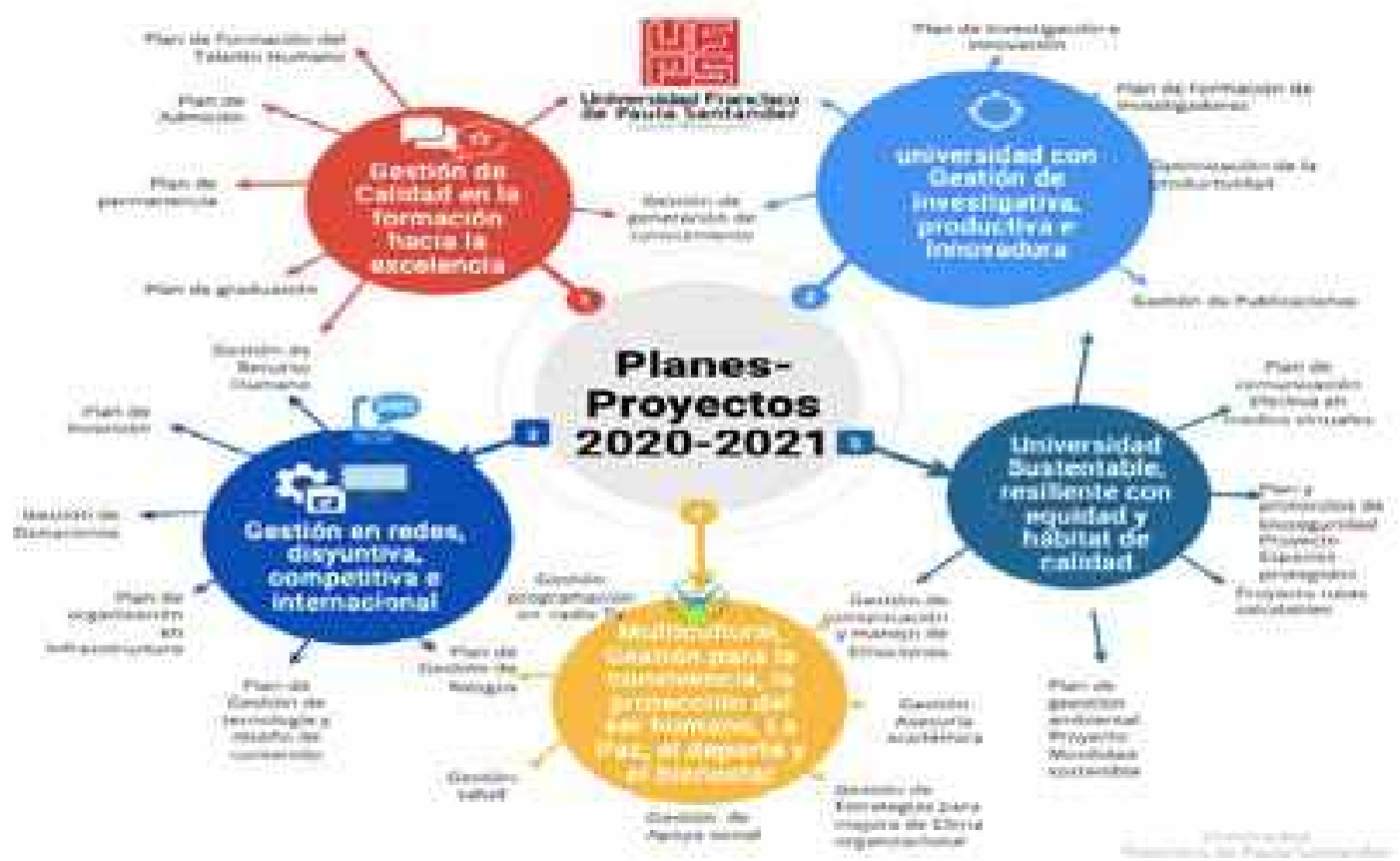

Figura 3. Planes Fuente: Autores

A31. Potenciar, conformar, gestionar y reestructurar los Institutos de investigación a fin de ser competitivos y visibles A32. Dinamizar la investigación capaz de proporcionar soluciones tecnológicas, econòmicas y sociales (captación de fondos sector privado, proteger y explotar responsablemente investigación, Fomentar publicaciones, Potenciar captación de proyectos, Validar cooperación entre grupos,Fomentar Banco de Patentes) A34. Incentivar el establecimiento de consorcios entre comunidad científica y empresa, para producir tecnología y la ecoinnovación, permitiéndoles producir productos y/o proporcionar servicios sostenibles. A35. Incentivar el registro de patentes y registros, A36. Diseñar Plan de Igualdad A37. Activar portal web para sensibilización, información y prevención de bullying, seguridad, bioetica, y reconocimiento A38. Diseño de programas dirigidos a la promoción de la inserción laboral, generaciòn de empresa A39.Implantar contabilidad analítica A40.Elaborar y gestionar plan anual de publicidad, estructurado, coherente y con combinación de medios y soportes, para la difusión integrada de la oferta A41.Posicionar y visualizar marcas y producciòn intelectual A42. Visibilidad de semilleros A43. Implantación efectiva de la administración electrónica en procesos de gestión A44. Racionalice el diseño del recurso humano, de manera que se permita su desarrollo profesional-personal, se incremente su grado de satisfacción, asegure la adecuación a objetivos y estrategias de la institución y capacidad de respuesta de las estructuras administrativas a las nuevas necesidades A45. Cumplir el equilibrio presupuestario dentro del modelo de financiación existente en convergencia de dicho modelo hacia niveles nacionales e internacionales. A46. Asegurar la autonomía financiera y eficiencia en el empleo de recursos en su prestación de servicios a la comunidad universitaria y a la sociedad. A48. Implantar estructuras administrativas eficientes y alineadas con la estrategia: Realizar proyectos para la mejora de la eficiencia de los edificios existentes e incorporar aspectos medioambientales como requisitos en proyectos de infraestructura A49. Impulsar relación estudiantes-egresados con el mercado laboral internacional A50.Incrementar la producción 
científica con visibilidad internacional en todos los campos y la promoción de las publicaciones de impacto y del número de proyectos competitivos internacionales de excelencia. A largo plazo continuar con formación de excelencia, alta Calidad, programas Académicos ambito nacional e internacional.

\section{CONCLUSIONES}

La universidad sustenta la oferta de sus programas educativos en un enfoque dialògico crítico, un modelo educativo pertinente, en permanente actualización, que fomenta la equidad, la formación integral de los estudiantes y el desarrollo y la asimilación de capacidades generales y especificas que permiten a sus egresados incorporarse y permanecer en los mundos laborales nacionales e internacionales, así como participar activamente en la evolución social y económica del País.

Surgen ejes estratègicos generación de cadena de valor con el saber y generaciòn de conocimiento, Identidad y, Universidad circular, para la comunidad, moderna e inteligente, con subejes Universidad con gestión de calidad hacia la excelencia, universidad con gestiòn investigadora, productiva e innovadora, universidad sustentable, resiliente con equidad y habitat de calidad, universidad multicultural con gestiòn para la convivencia, la protecciòn del ser humano, la paz, el deporte y el bienestar; con gestiòn en redes, disruptiva, competitiva e internacional.

\section{REFERENCIAS BIBLIOGRÁFICAS}

Arboleda, N. (2007). El perfil socioeconómico de egresados en programas de educación superior virtual. Bogotá: Corporación Unificada Nacional de Educación Superior.

Beltrán J 2012 Guía para una gestión basada en procesos (España: IAT).

Bedoya, D. (2011). Elementos de calidad de la educación superior en Colombia. Caso de Estudio: Universidades 2009-2011. Recuperado de http://www mercadeo/ benchmarking $\% 202009 \% 20$ ies $\% 20$ pag\%2030.html.

Castilla-Duarte, M, Jácome-Carrascal, J y Vergel Ortega, M. (2020), Emprendemat, su influencia en el desarrollo de competencias económica-financieras en la juventud de la región del Catatumbo, Aibi revista de investigación, administración e ingeniería: Vol. 8, Núm. 1

DANE (2018). Censo nacional adelantado por el Departamento Administrativo Nacional de Estadística

Bernal Fonseca, N., Muñoz, C., y Vergel. M. (2018). Fomento de la convivencia en instituciones de educación superior. Revista Logos 10(1), 68-83.

Freire, P. (1997). Pedagogia da Esperança: Um reencontro com a Pedagogia do oprimido. $4^{a}$ ed. (1ª edición: 1992). Rio de Janeiro: Paz e Terra

Largo-Leal, Y. Vergel-Ortega, M., Rojas, J. (2020). Empatìa Bogotà: ECOE Ediciones

Parra, H., Vergel Ortega, M., \& Martínez, J. (2013). Metodología para elaborar planes de capacitación en instituciones de Educación superior. Revista Logos Ciencia \& Tecnología, 5(1), 205-213. doi: http:// dx.doi.org/10.22335/rlct.v5i1.388

Orjuela, J.L, Gómez, C.S. y Vergel, M. 2020. Desarrollo de competencias de innovación en estudiantes de cálculo a traves de proyectos de creación en tiempos de asesoria en casa. Respuestas. 9(5). 
Vergel, M. \& Gallardo, H. (2007). Modelación en un museo interactivo. Recuperado en: http://www.cientec.or.cr/pop/2007/COMawencyVergel.pdf

Vergel Ortega, M., Zafra Tristancho, S.L., Parra López, H.M. Modelo para evaluar la pertinencia de programas en educación superior (2018). Alemania: Editorial Académica Española ISBN: 978-620-213104-9 